\title{
Minor effect of depletion of resident macrophages from peritoneal cavity on resistance of common carp Cyprinus carpio to blood flagellates
}

\author{
Jeroen P. J. Saeij ${ }^{1}$, Adrie Groeneveld ${ }^{1}$, Nico Van Rooijen ${ }^{2}$, Olga L. M. Haenen ${ }^{3}$, \\ Geert F. Wiegertjes ${ }^{1, *}$
}

${ }^{1}$ Cell Biology and Immunology Group, Wageningen Institute of Animal Sciences, Wageningen University, PO Box 338, 6700 AH Wageningen, The Netherlands

${ }^{2}$ Free University, Department of Molecular Cell Biology, Faculty of Medicine, POB 7057, 1007 MB Amsterdam, The Netherlands ${ }^{3}$ Central Institute for Animal Disease Control, POB 2004, 8203 AA Lelystad, The Netherlands

\begin{abstract}
Carp Cyprinus carpio macrophages were depleted by intraperitoneal (i.p.) injection of clodronate-liposomes for the in vivo study of the effect of macrophage depletion on the resistance of carp to infection with blood flagellate parasites. Clodronate released inside the cell induces apoptosis of (murine) macrophages. Following i.p. injection of carp with liposomes alone, but not with Trypanoplasma borreli, neutrophilic granulocytes rapidly migrated from the head kidney to the peritoneal cavity. The majority of liposomes in the peritoneal cavity were not taken up by newly arrived neutrophilic granulocytes, however, but by resident macrophages. After 2 i.p. injections of clodronate-liposomes, the percentage of macrophages present in the peritoneal cavity was significantly reduced, as evaluated by flow cytometry. Macrophage-depleted carp that were infected i.p. with $T$. borreli suffered from high mortality. However, these fish did not show lethal parasitaemia but did show clear bacteraemia. Macrophage-depleted carp that were infected i.p. with Trypanosoma carassii showed a minor increase in parasitaemia. In addition, macrophage-depleted carp, immune to T. borreli as a result of having survived a prior infection, remained immune to i.p. reinfection with T. borreli. Succesful depletion of peritoneal macrophages seemed to have a minor effect on the resistance of carp against blood flagellates. However, carp macrophages are essential as a first line of defence against (bacterial) infection.
\end{abstract}

KEY WORDS: Cyprinus carpio $\cdot$ Macrophages $\cdot$ Clodronate $\cdot$ Trypanoplasma borreli $\cdot$ Trypanosoma carassii

Resale or republication not permitted without written consent of the publisher

\section{INTRODUCTION}

In vivo functions of (rodent) macrophages can be studied by the liposome-mediated macrophage-depletion model (Van Rooijen \& Van Nieuwmegen 1984). Injected liposomes containing dichloromethylene-bisphosphonate (clodronate) are taken up by macrophages in which clodronate is released following uptake into phagosomes and disruption of phospholipid bilayers. The released clodronate induces apoptosis in macrophages only (Van Rooijen et al. 1985, 1996). For fish, the macrophage-depletion model has only been applied to study macrophage function in rainbow trout Oncorhynchus mykiss. Espenes et al. (1997) observed that clodronate-liposome treatment resulted in a reduced activity of macrophage-specific acid phosphatase in the spleen, and found ultrastructural evidence for a large increase in the number of apoptotic bodies. Although they concluded that the model could prove useful for investigating the function of piscine macrophages in vivo, so far no functional studies have been reported. The aim of the present study was to develop the macrophage-depletion model for carp and to use this model to study, in vivo, the 
effect of macrophage depletion on the resistance of carp to infection with blood flagellates.

Trypanoplasma (syn. Cryptobia) borreli and Trypanosoma carassii (syn. T. danilewskyi) are leechtransmitted protozoan parasites of cyprinid fishes. Both parasites ('tryps') are in the order Kinetoplastida but belong to different suborders (Bodonina and Trypanosomatina, respectively). Although the parasites are evolutionarily distant, mixed infections with $T$. borreli and $T$. carassii are widespread in farmed populations of cyprinids (Lom \& Dykova 1992). Whilst in aquaculture, as in nature, blood-sucking leeches act as vectors for transmitting 'tryps' between fishes, laboratory infections are usually achieved by syringe-passage, allowing for careful experimentation on fishes (Jones et al. 1993). In these experimental infections, when carp are injected intraperitoneally (i.p.) with $T$. borreli, parasites multiply locally at the site of injection during the initial lag phase (Steinhagen et al. 1989) and appear in the blood 1 to 2 wk after infection. Finally, after several weeks, fish with high parasitaemia show a severe destruction of kidney tissue, which is the probable cause of death (Bunnajirakul et al. 2000). These carp, unable to limit initial multiplication, are overwhelmed by rapidly multiplying parasites, and parasitaemia increases to lethal concentrations of 1 to $2 \times 10^{8} \mathrm{ml}^{-1}$ blood (Wiegertjes et al. 1995). In these fish, it is possible that resident (peritoneal) leukocytes fail to limit the initial multiplication of trypanoplasms, thereby allowing for a rapid multiplication of parasites. To investigate the effect of a depletion of peritoneal macrophages on the resistance of carp to blood flagellates we treated carp with intraperitoneal injections of clodronate-liposomes and studied parasitaemia after subsequent infection.

\section{MATERIALS AND METHODS}

Fish. Parasite-free carp Cyprinus carpio were the offspring of a cross between fish of Hungarian origin (R8 strain) and of Polish origin (R3 strain) (Irnazarow 1995). Carp were propagated and raised under parasite-free conditions in the central fish facility (Wageningen) at $23 \pm 2^{\circ} \mathrm{C}$ in recirculating UV-treated tap water. Initially, fish larvae were fed with Artemia sp. nauplii (hatched from Sanders Great Salt Lake Artemia sp. cysts); subsequently (after 3 to $4 \mathrm{wk}$ ) the feeding regime was switched to commerical pelleted dry food for carp (Trouw, Nutreco). Carp used in the experiments were sexually immature and had a body weight of approx. $150 \mathrm{~g}$. For in vitro experiments, carp were directly taken from the water recirculation system and killed for dissection. For the infection experiments, carp were kept in a quarantine unit at least
1 mo prior to infection for acclimation. For these infection experiments, fish were equally divided over 10 (Trypanoplasma borreli infection experiment) or 4 (Trypanosoma carassii infection experiment) glass tanks with filtered and UV-treated recirculating water at $20 \pm 2^{\circ} \mathrm{C}$ (see also Table 1 ).

Preparation of liposomes. Liposomes were prepared as described by Van Rooijen \& Sanders (1994). Briefly, $86 \mathrm{mg}$ phosphatidylcholine and $8 \mathrm{mg}$ cholesterol were dissolved in chloroform in a round-bottomed flask. The thin film formed against the wall of the flask by vacuum evaporation at $37^{\circ} \mathrm{C}$ was dispersed by gentle rotation for $10 \mathrm{~min}$ in $10 \mathrm{ml} 0.6 \mathrm{M}$ dichloromethylenebisphosphonate (clodronate; Roche Diagnostics) solution (clodronate-liposomes) or in $10 \mathrm{ml}$ phosphatebuffered saline (PBS), pH 7.4 (PBS-liposomes). Liposomes were also labelled with the fluorescent lipophilic carbocyanine dye 1,1'-dioctadecyl-3, 3, 3', 3'tetramethylindocarbocyanine perchlorate (DiI) by adding $5 \mu l 2.5 \mathrm{mg} \mathrm{ml}^{-1}$ DiI to $100 \%$ ethanol (DiI-liposomes). Suspensions were kept at room temperature for $2 \mathrm{~h}$ under nitrogen gas and sonicated for $3 \mathrm{~min}$. After another $2 \mathrm{~h}$, free clodronate was removed by centrifugation $(25000 \times g, 20 \mathrm{~min})$. Liposomes were washed in PBS, centrifuged, resuspended in $4 \mathrm{ml}$ PBS and stored at $4^{\circ} \mathrm{C}$.

Isolation of cells for flow cytometry. To collect leukocytes from naïve individuals, carp were killed for dissection by an overdose of tricaine methane sulfonate (TMS; Crescent Research Chemicals) buffered with sodium bicarbonate (Sigma). Blood was collected and diluted 1:1 in RPMI-1640 (Sigma) adjusted to

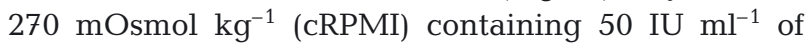
heparin (Leo Pharmaceutical Products). After centrifugation at $100 \times g$ for $10 \mathrm{~min}$, the leukocyte-containing supernatant was collected and layered on a 1.02 to 1.083 Percoll gradient. Cell suspensions from spleen, liver and head kidney were prepared by passing the organs through a $50 \mu \mathrm{m}$ nylon mesh using the barrel from a $10 \mathrm{ml}$ syringe. Leukocytes from the peritoneal cavity were collected by peritoneal lavage. Total cell fractions were isolated using a 1.02 to 1.083 Percoll gradient to remove erythrocytes (density $>1.083$ ) and debris/fat (density < 1.02) $(100 \%$ Percoll density $1.130 \mathrm{~g} \mathrm{ml}^{-1}$; Amersham Pharmacia Biotech).

Flow cytometry. Fluorescence and cell characteristics were measured by flow cytometry. Briefly, cells (5 $\times 10^{5}$ ) were washed in cRPMI with $1 \% \mathrm{w} / \mathrm{v}$ bovine serum albumin (BSA) and incubated with the first antibody for $0.5 \mathrm{~h}$ on ice. After washing, cells were incubated with the second antibody, rabbit-anti-mouse(R)-phycoerythrin (PE) (RAM-RPE， 1:100 v/v; Dako A/S) or RAM-RPE-cy5 (1:50 v/v; Dako A/S) for $0.5 \mathrm{~h}$ on ice, washed, and analysed by flow cytometry. For flow cytometric analysis, data for $10^{4}$ counts were collected 
using a FACStar flow cytometer (Beckton-Dickinson, single excitation wavelength of $488 \mathrm{~nm}$ ). Forward scatter (FSC) and side scatter (SSC) characteristics of $10^{4}$ events were acquired in linear mode; fluorescence intensities were acquired on a log scale.

We used a combination of cell type-specific markers and FSC/SSC profiles in flow cytometry to study leukocyte redistribution after clodronate-liposome treatment. Neutrophilic granulocytes were labelled with monoclonal antibody TCL-BE8 (1:50) (Nakayasu et al. 1998). At this dilution, TCL-BE8 binds to monocytes with low affinity only (J.H.W.M. Rombout unpubl. data). B-lymphocytes (low FSC/SSC) were labelled with monoclonal antibody WCI-12 (1:50) reacting against carp immunoglobulin, Ig (Secombes et al. 1983). Macrophages (high FSC/SSC, TCL-BE8', WCI$12^{-}$) could be distinguished from neutrophilic granulocytes (high FSC/SSC, TCL-BE8 ${ }^{+}$, WCI-12-), B-lymphocytes (low FSC/SSC, TCL-BE8-, WCI-12+) and T-lymphocytes (low FSC/SSC, TCL-BE8 ${ }^{-}$, WCI-12-).

Apoptosis was measured by labelling exposed phosphatidylserine of cells in incubation buffer $(10 \mathrm{mM}$ HEPES, $140 \mathrm{mM} \mathrm{NaCl}, 5 \mathrm{mM} \mathrm{CaCl}_{2}, 270 \mathrm{mOsm} \mathrm{kg}^{-1}$ ) using Annexin- $\mathrm{V}$ conjugated with fluorescein isothiocyanate (FITC) or phycoerythrin ( $\mathrm{PE}$; Boehringer). The percentage of necrotic cells was determined by propidium iodide (PI) staining.

Cytofluorometric analyses. Murine macrophages can be depleted by injecting clodronate-containing liposomes that are taken up by macrophages, resulting in release of clodronate following disruption of the phospholipid bilayers of phagosomes (Van Rooijen \& Van Nieuwmegen 1984). We examined the use of this macrophage-depletion model for carp. Flow cytometric experiments were performed at least in duplicate on at least 2 independent occasions as follows:

- Leukocyte redistribution in response to parasitetreatment alone was studied $16 \mathrm{~h}$ after i.p. injection of Trypanoplasma borreli $\left(2.5 \times 10^{3}\right.$ in $\left.100 \mu \mathrm{l}\right)$ or PBS $(100 \mu \mathrm{l})$.

- Leukocyte redistribution in response to liposomes alone was studied $16 \mathrm{~h}$ after i.p. injection of $0.5 \mathrm{ml}$ fluorescent (DiI)-liposomes.

- Leukocyte redistribution in response to clodronate treatment was studied after i.p. injection of $0.5 \mathrm{ml}$ clodronate-liposomes, PBS-liposomes or PBS on Days -4 and -2 .

- Residual macrophage function (uptake of liposomes) after clodronate treatment was studied following i.p. injection of $0.5 \mathrm{ml}$ clodronate-liposomes, PBSliposomes or PBS 4 and $2 \mathrm{~d}$ prior to analysis, i.e. on Days -4 and -2 . To study residual uptake of liposomes, fish were i.p.-injected with $0.5 \mathrm{ml}$ fluorescent (DiI)liposomes $16 \mathrm{~h}$ prior to measurement, i.e. $16 \mathrm{~h}$ before Day 0.
Parasite infection. Trypanoplasma borreli was cloned and characterised by Steinhagen et al. (1989) and maintained by syringe passage through laboratory-bred carp. Trypanosoma carassii was cloned and characterised by Overath et al. (1998), named Clone $\mathrm{K} 1$, and maintained by in vitro culture. For blood sampling, carp were anaesthetised in $0.3 \mathrm{~g} \mathrm{l}^{-1} \mathrm{TMS}$ buffered with $0.6 \mathrm{~g} \mathrm{l}^{-1}$ sodium bicarbonate (Sigma). Blood was collected by vena puncture of the caudal vessel. We diluted $25 \mu \mathrm{l}$ of blood 10 times in cRPMI medium containing $50 \mathrm{IU} \mathrm{ml}^{-1}$ heparin for counting parasites. Parasitaemia was monitored using a Bürker counting chamber (minimal detection limit $10^{5}$ parasites $\mathrm{ml}^{-1}$ blood).

Effect of clodronate-liposome treatment on parasitaemia. Naïve fish $(\mathrm{n}=120$ per infection experiment, see Table 1) were randomly allocated to 3 groups of 40 , numbered by tattoo, and subjected to 1 of 3 treatments: clodronate-liposomes, PBS-liposomes, or PBS only. Each aquarium contained representatives in equal numbers of all 3 treatment groups $\left(n=12\right.$ aquarium $^{-1}$ for Trypanoplasma borreli, $\mathrm{n}=30$ aquarium $^{-1}$ for Trypanosoma carassii infection studies). We removed 2 carp per group at weekly intervals for histological analysis. Moribund fish were examined for the presence of parasites and for the presence of bacteria.

The effect of clodronate treatment on carp resistance to Trypanoplasma borreli was studied by i.p. injection of carp with $0.5 \mathrm{ml}$ clodronate-liposomes, PBSliposomes or PBS on Days -2, 7, 14 and 21. On Day 0, carp were i.p.-injected with $2500 \mathrm{~T}$. borreli or with PBS $(100 \mu \mathrm{l})$. Blood samples were taken at weekly intervals post-infection for determination of parasitaemia.

The effect of clodronate treatment on carp resistance to Trypanosoma carassii was studied by i.p. injection of carp with $1 \mathrm{ml}$ clodronate-liposomes, PBS-liposomes or PBS on Days -4 and -2 . In this experiment, the treatment frequency and dose for clodronate-liposome injections (and the corresponding controls) were slightly adjusted compared with the experiment described above because the clodronate treatment applied to the Trypanoplasma borreli infections resulted in relatively high numbers of moribund fish. In a pilot experiment, 1 to $1.5 \mathrm{ml}$ was found to be the maximum dose of clodronate-liposomes that did not produce clinical effects. On Day 0, carp were i.p.-injected with $5 \times 10^{4} \mathrm{~T}$. carassii or with PBS $(100 \mu \mathrm{l})$. The infection dose for $T$. carassii was higher than that for $T$. borreli because carp are more resistant to the former than to the latter (Overath et al. 1999). Blood samples were taken at weekly intervals post-infection for determination of parasitaemia.

Carp surviving a Trypanoplasma borreli infection are protected against reinfection for more than $1 \mathrm{yr}$ (G. F. Wiegertjes unpubl. data). The nature of this pro- 
tective immunity is unknown. The effect of clodronate treatment on carp refractory to reinfection with $T$. borreli was studied by treating fish with large volumes of clodronate-liposomes. Nine immune carp (survivors from an infection $1 \mathrm{yr}$ earlier) were i.p.- and intravenously (i.v.)-injected with 1.5 and $0.5 \mathrm{ml}$, respectively, of clodronate-liposomes ( $\mathrm{n}=3$ ), PBS-liposomes $(n=3)$ or PBS $(n=3)$ on Days -4 and -2 . On Day 0, carp were i.p.-injected $(100 \mu \mathrm{l})$ with a high dose $\left(10^{6}\right)$ of $T$. borreli. Blood samples were taken at weekly intervals for determination of parasitaemia. As a control, naïve fish of the same group $(n=3)$ were also infected with $T$. borreli.

Bacteriology. Routine bacteriology was performed when there were clinical signs of disease. As a control, apparently healthy fish were also sampled for bacteriology. Sterile cotton-swab samples were taken from skin lesions, ascites, and internal organs (spleen and/or kidney). The swab specimens were inoculated onto brain heart infusion (BHI) agar with $5 \%$ v/v sheep blood, and incubated at $22^{\circ} \mathrm{C}$ for at least $5 \mathrm{~d}$. Additionally, in case of skin lesions, specimens from these lesions were inoculated simultaneously onto Cytophaga Agar (Oxoid). Bacterial growth was identified according to standard methods (Krieg \& Holt 1984, Austin \& Austin 1987, Barrow \& Feltham 1993).

Statistical methods. Significance of differences was determined by Student's $t$-test. When multiple comparisons were made with a single control, a Bonferroni correction was used to adjust the observed significance level. Differences were considered to be significant at $\mathrm{p}<0.05$.

\section{RESULTS}

\section{Effect of parasite injection on leukocyte redistribution}

We decided to study the effect of macrophage depletion in the peritoneal cavity. Although i.p. injection, in contrast to intramuscular injection, may not be considered a natural route of infection for trypanosomes, it is frequently used to establish experimental infections (Jones et al. 1993). Also, i.p. injection greatly facilitated the collection of leukocytes for studies on the effect of (clodronate)-liposome treatments on leukocyte redistribution.

First we studied the redistribution of leukocytes in the peritoneal cavity after i.p. injection with parasites (Trypanoplasma borreli). Leukocytes were washed from the peritoneal cavity $16 \mathrm{~h}$ post-injection and cell types defined with the flow cytometer on the basis of cell-specific markers and FSC and SSC characteristics. The composition of the peritoneal leukocyte cell popu- lation after injection of $T$. borreli was identical to that of control fish injected with PBS. The majority of cells $(65 \pm 11 \% \mathrm{SD})$ were identified as macrophages, the other cell types being neutrophils $(19 \pm 10 \%)$ and lymphocytes $(17 \pm 6 \%)$. Thus, experimental i.p. infection with $T$. borreli does not lead to a rapid redistribution of leukocytes. Most probably, the major cell type encountered by $T$. borreli when injected i.p. is carp macrophages.

\section{Effect of liposome injection on leukocyte redistribution}

Having characterized the resident pool of leukocytes in the peritoneal cavity, we investigated the effect of i.p. injection of liposomes, which is an essential part of the macrophage depletion method. Leukocyte cell types were defined with the flow cytometer on the basis of cell-specific markers and FSC and SSC characteristics. In fish injected with fluorescent (DiIloaded) liposomes alone, neutrophilic granulocytes migrated from the head kidney (decrease from $38 \pm 7$ to $14 \pm 5 \%$ ) to the peritoneal cavity (increase from $19 \pm$ 10 to $70 \pm 15 \%$ ). This resulted in a 20 -fold increase (from $0.5 \pm 4 \times 10^{6}$ to $10 \pm 6 \times 10^{6}$ ) in total numbers of leukocytes in the peritoneal cavity wash that could mainly be attributed to newly arrived neutrophils. The total number of cells was determined by concentrating all cells in $1 \mathrm{ml}$ and calculated using a haemocytometer. No significant influx of macrophages was observed.

At $16 \mathrm{~h}$ after injection of liposomes, both fluorescent (DiI-positive) neutrophilic granulocytes and macrophages were seen. Macrophages, although lower in total numbers, showed more intense fluorescence (mean fluorescence intensity $288 \pm 85$ SD) than neutrophilic granulocytes (mean fluorescence intensity $80 \pm 25$ ), and the difference was highly significant. Thus, although both macrophages and newly arrived neutrophilic granulocytes apparently took up liposomes, the difference in fluorescence suggested that macrophages took up a much higher number of liposomes per cell than neutrophilic granulocytes. This supported the use of the liposome carrier for a depletion targeted towards carp macrophages.

\section{Effect of clodronate-liposome injection on leukocyte redistribution}

Intraperitoneal injection with liposomes alone resulted in a migration of neutrophilic granulocytes, but not macrophages, to the peritoneal cavity. We then examined the effect of i.p. injection of clodronate- 


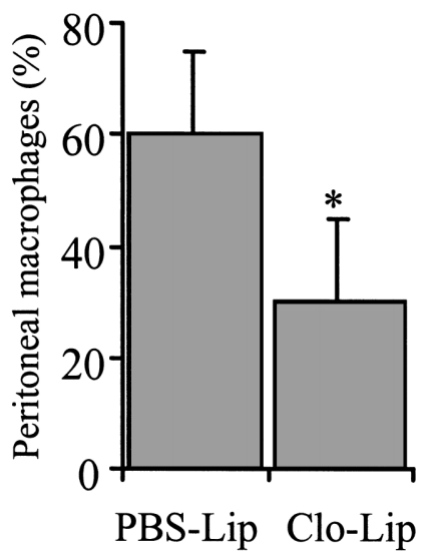

Fig. 1. Cyprinus carpio. Effect (mean $+\mathrm{SD}$ ) of clodronateliposome injection on carp peritoneal macrophages. Carp $(\mathrm{n}=2$ ) were injected twice (Days -4 and -2 ) with $0.5 \mathrm{ml}$ clodronate-liposomes (Clo-Lip) or PBS-liposomes (PBS-Lip). Macrophages were defined as TCL-BE8 $8^{-}$and WCI-12- cells with a high forward scatter/side scatter in flow cytometry. Total number of leukocytes was the same for both liposome treatments. This is 1 representative experiment out of 2 independent experiments with similar results. *Significantly different from PBS-Lip-treated control $(p<0.05)$

containing liposomes (Days -4 and -2 ). The percentage of peritoneal macrophages on Day 0 (60 $\pm 15 \%$, Fig. 1) was similar to that analysed $16 \mathrm{~h}$ after PBS injection ( $65 \pm 11 \%$, see first subsection of 'Results'), suggesting that putative populations of neutrophilic granulocytes, newly arrived in the peritoneal cavity because of PBSliposome injections, were no longer present. The clodronate-liposome treatment significantly lowered the percentage of peritoneal macrophages compared with PBS-liposome-treated carp (Fig. 1).

In the head kidney as well, neutrophilic granulocytes had returned to normal values of approximately $40 \%$ at Day 0 following clodronate-liposome treatment. This was probably the result of regeneration of new cells from (head) kidney precursor cells, since the FSC/ SSC characteristics of the (TCL-BE8 ${ }^{+}$) neutrophils in the head kidneys of liposome-treated fish differed (smaller/rounder) from those in naïve (PBS-treated) fish. Clodronate-liposome treatment did not result in a notable redistribution of leukocytes from the spleen, liver or blood (data not shown).

\section{Effect of clodronate-liposome injection on residual macrophage function}

Having established that primarily macrophages took up (fluorescent) liposomes and that i.p. injection of clodronate-liposomes specifically affected peritoneal macrophages, we proceeded to study the residual macrophage function (uptake of liposomes) in fish treated with clodronate-liposomes. Fish treated with clodronate-liposomes as above (i.p. Days -4 and -2 ) and the corresponding controls were also injected with fluorescent liposomes (i.p. Day -1). The effect of prior clodronate-liposome treatment on the ability of leukocytes to take up fluorescent (DiI)-liposomes was evaluated on Day 0. Prior clodronate-liposome treatment did not significantly affect the percentage of DiIpositive leukocytes in the peritoneal cavity, compared with prior PBS-liposome treatment (Fig. 2). However, the fluorescence intensity in peritoneal leukocytes collected from carp treated with clodronate-liposomes was significantly lower than in the corresponding control, again suggestive of a primary effect on macrophages (Fig. 3). The effect of prior clodronate-liposome treatment on the percentage of DiI-positive leukocytes in immune organs such as spleen, liver and head kidney was also examined and compared with prior PBSliposome treatment. A slight but significant reduction in the percentage of cells that had taken up DiI-liposomes was observed in the liver, but not in other organs examined (Fig. 2).

\section{Effect of clodronate-liposome treatment on resistance to Trypanoplasma borreli}

To study the effect of clodronate-liposome treatment on resistance to Trypanoplasma borreli, carp were randomly allocated to 3 groups, each of which received

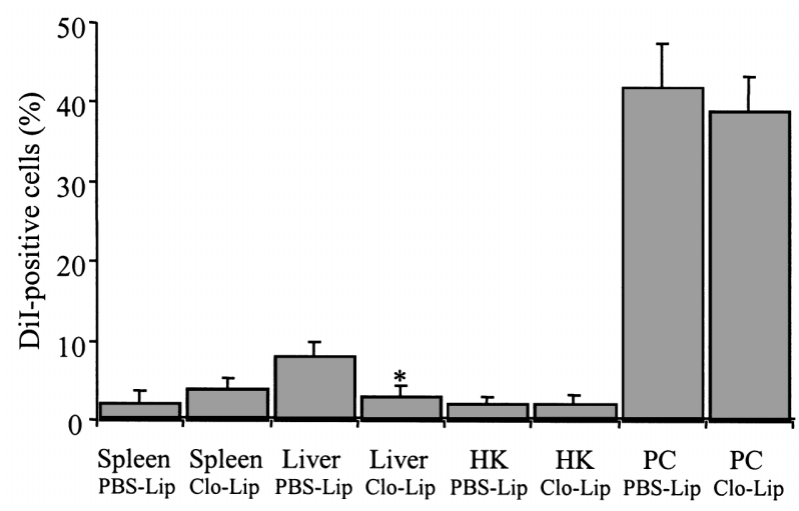

Fig. 2. Cyprinus carpio. Effect of prior clodronate-liposome treatment on the ability to take up DiI-liposomes, showing percentage (mean $+\mathrm{SD}$ ) of DiI-positive leukocytes in spleen, liver, head kidney (HK) and peritoneal cavity (PC). Carp $(\mathrm{n}=2)$ were injected twice (Days -4 and -2 ) with $0.5 \mathrm{ml}$ clodronate-liposomes (Clo-Lip) or PBS-liposomes (PBS-Lip) followed by i.p. injection (Day -1) of DiI-liposomes. Fluorescence of positive cells was measured with flow cytometry (Day 0). This is 1 representative experiment out of 2 independent experiments with similar results. *Significantly different from PBS-Lip-treated control $(\mathrm{p}<0.05)$ 


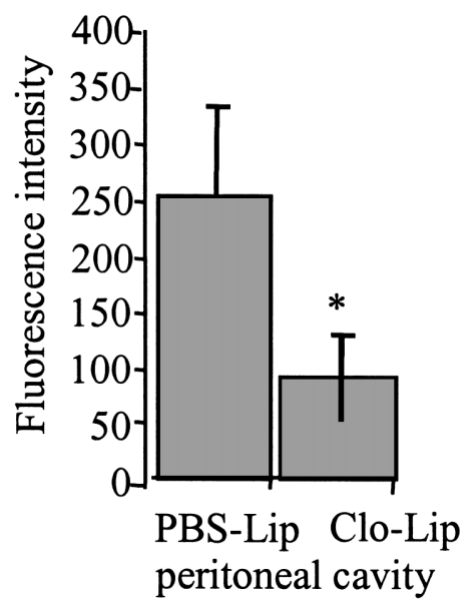

Fig. 3. Cyprinus carpio. Effect of prior clodronate-liposome treatment on the ability to take up DiI-liposomes. Fluorescence intensity (relative units, mean $\pm \mathrm{SD}$ ) of DiI-positive cells in the peritoneal cavity. Further details as in legend to Fig. 2

a different treatment (clodronate-liposomes, PBSliposomes, PBS) and were i.p.-injected with $T$. borreli or with PBS on Day 0 (Table 1). Infection of each individual fish was confirmed by parasite counts and no parasites were recovered at any time from non-infected carp. Parasitaemia at $14 \mathrm{~d}$ post infection (p.i.) was in the range of the lower detection limit $\left(1 \times 10^{5}\right)$. Parasite counts at 14 and $21 \mathrm{~d}$ p.i. showed no effect of clodronate-liposome treatment (Fig. 4). Neither was an effect of PBS-liposome treatment observed. At Days 28 and 35 p.i., fish treated with clodronate-liposomes showed slightly higher parasitaemia than control (PBS-liposomes or PBS) fish. The increase in parasitaemia was almost significant $(p<0.09$ and $\mathrm{p}<0.065$, respectively).

From the infected clodronate-treated group, moribund carp were removed from Day 19 p.i. onwards, and cumulative mortality recorded at Day 40 p.i. was $85 \%$ (Fig. 5). However, moribund carp were not necessarily heavily infected with parasites. Although some of these carp sometimes had high parasitaemia, no clear relation existed between parasitaemia and survival time (only 2 fish) had approximately $0.8 \times 10^{8}$ $\mathrm{ml}^{-1}$, whereas 1 to $2 \times 10^{8} \mathrm{ml}^{-1}$ is considered lethal). In

Table 1. Cyprinus carpio. Experimental set-up for infection studies. Per treatment group, fish were equally divided among 4 (Trypanoplasma borreli) or 10 (T. carassii) glass tanks with filtered and UV-treated recirculating water at $20 \pm 2^{\circ} \mathrm{C} . \mathrm{n}=$ number of fish

\begin{tabular}{|lccc|}
\hline & $\begin{array}{c}\text { Clodronate-liposomes } \\
\text { (n) }\end{array}$ & $\begin{array}{c}\text { PBS-liposomes } \\
\text { (n) }\end{array}$ & $\begin{array}{c}\text { PBS } \\
\text { (n) }\end{array}$ \\
\hline Infected & 30 & 30 & 30 \\
Non-infected & 10 & 10 & 10 \\
\hline
\end{tabular}

fact, more than $50 \%$ of the moribund fish showed relatively low parasitaemia $\left(<5 \times 10^{6} \mathrm{ml}^{-1}\right)$. All moribund individuals, however, showed ascites and enlarged spleens with multibacterial infections. Aeromonas hydrophila and A. sobria were most often identified. Control carp that received the same treatment with clodronate-liposomes but were not infected with parasites also showed signs of heavy bacterial infection. Although this could be observed in only a few (4 of 10)

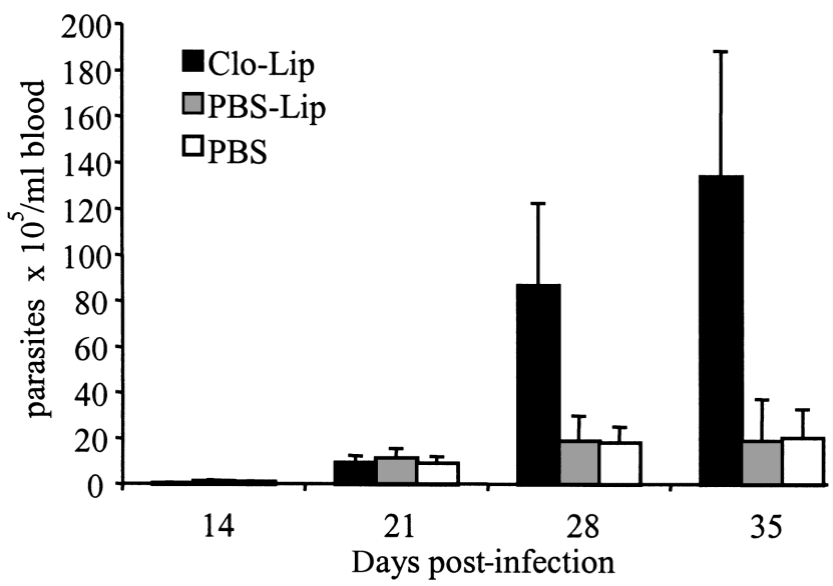

Fig. 4. Cyprinus carpio. Effect (mean + SD) of clodronateliposome treatment on resistance to Trypanoplasma borreli. Carp were treated (0.5 ml i.p., Days -2, 7 and 14) with clodronate-liposomes (Clo-Lip, $\mathrm{n}=30$ ), PBS-liposomes (PBSLip, $n=30)$ or PBS $(n=30)$. Differences in parasitaemia between groups were not statistically significant at $\mathrm{p}<0.05$

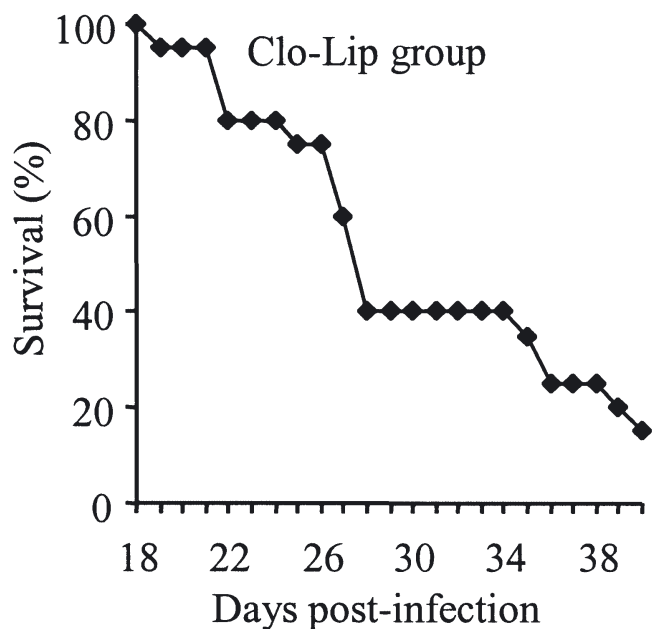

Fig. 5. Cyprinus carpio. Effect of clodronate-liposome treatment on resistance to Trypanoplasma borreli. Carp were treated $(0.5 \mathrm{ml}$ i.p., Days $-2,7$ and 14$)$ with clodronate-liposomes (Clo-Lip, $\mathrm{n}=30$ ), PBS-liposomes (PBS-Lip, $\mathrm{n}=30$ ) or with PBS $(\mathrm{n}=30)$. Only clodronate-treated carp died. There was $100 \%$ survival of PBS-Lip- and PBS-injected fish (data not shown) 
control carp, since the other (6 of 10) control carp had been sacrificed for histology early during the experiment, this suggested that the multibacterial infections could not be primarily ascribed to co-infection with parasites and were likely to have been caused by the clodronate-liposome treatment.

\section{Effect of clodronate-liposome treatment on resistance to Trypanosoma carassii}

To study the effect of clodronate-liposome treatment on resistance to Trypanosoma carassii a similar experimental design was used. However, as weekly injections with clodronate-liposomes apparently led to increased infections with common bacteria, we chose to treat carp only twice with clodronate-liposomes (Days -4 and -2 ). Parasite infection was confirmed for each infected individual fish but not for uninfected individuals. Parasitaemia at Day 11 p.i. was significantly $(p<0.013)$ higher in fish treated with clodronate-liposomes than in control (PBS or PBSliposomes) fish. However, from Day 19 p.i. onwards, parasitaemia in the clodronate-liposome-injected group was not significantly different from parasitaemia in the control groups (Fig. 6). No mortality was observed and parasitaemia returned to below the lower detection limit at $80 \mathrm{~d}$ p.i.

\section{Effect of clodronate-liposome treatment on fish immune to Trypanoplasma borreli}

Carp surviving a Trypanoplasma borreli infection are protected against reinfection for more than $1 \mathrm{yr}$. In order to thoroughly deplete macrophages, we injected a large dose of clodronate-liposomes on Days -4 and -2 before infection. Control carp received PBS-liposomes or PBS only. On Day 0, macrophage-depleted carp and the corresponding controls were i.p.-injected with a high dose of $T$. borreli, while non-immune carp from the same initial experiment were also infected. None of the immune carp showed parasites at Days 7 , 14 and 21 p.i. One of the clodronate-liposome-treated carp died at Day 5 p.i., apparently because of heavy bacteraemia (no parasites were seen). All infected nonimmune carp had high parasitaemia (data not shown).

\section{DISCUSSION}

In this study, i.p. treatment with liposome-encapsulated clodronate was used to selectively deplete peritoneal carp macrophages in vivo. In mice, i.p. administration of liposomes enables complete elimination of

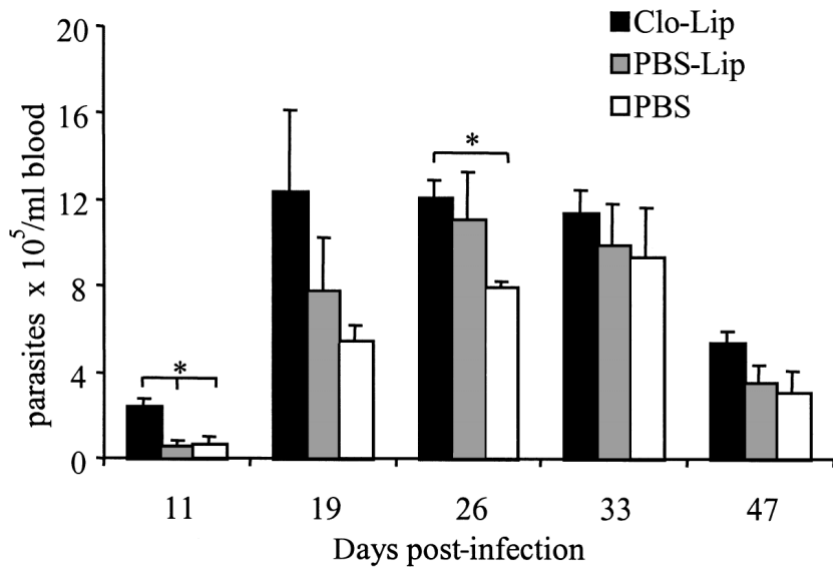

Fig. 6. Cyprinus carpio. Effect (mean $+\mathrm{SD}$ ) of clodronateliposome treatment on resistance of carp to Trypanosoma carassii. Carp were treated (1 ml i.p., Days -4 and -2$)$ with clodronate-liposomes (Clo-Lip, $\mathrm{n}=30$ ), PBS-liposomes (PBSLip, $\mathrm{n}=30$ ) or with PBS ( $=30)$. *Significantly different from controls $(\mathrm{p}<0.05)$

macrophages from the peritoneal cavity in $2 \mathrm{~d}$ (Biewenga et al. 1995). In mice, clodronate-liposomes are taken up to a significant extent by macrophages only, leading to their apoptosis (Van Rooijen et al. $1985,1996)$. We also tried to evaluate the effects of clodronate-liposome treatment on carp macrophages by measuring apoptosis, but did not succeed in demonstrating significant differences between treatment groups (data not shown). We used Annexin V-labelling at Days 1 and 2 after clodronate-liposome treatment to measure apoptosis. It is not unlikely that we have missed a putative effect of clodronate-liposome treatment on apoptosis since, in mammals, apoptotic cells are rapidly removed (Henson et al. 2001). The only study so far that reported the use of this model in fish (rainbow trout Oncorhynchus mykiss) did report more apoptotic cells in clodronate-treated fish as measured by TUNEL (Tdt-mediated dUTP nick-end labelling; Espenes et al. 1997). Espenes et al. (1997) also showed, using acid phosphatase staining of frozen tissuesections, that rainbow trout phagocytic cell populations were depleted close to the sinusoids in head kidney and spleen and in the splenic ellipsoids, apparently consistent with the targeting of liposomes to macrophages. We also sampled carp at regular intervals for similar histological analysis of frozen tissuesections, but have chosen not to present these results. Although we could clearly identify acid phosphatasepositive cells in spleen, liver and head kidney, we found the method, at least in carp, unreliable for an unambiguous demonstration of differences in macrophage cell numbers after clodronate-liposome treatment. Therefore, we chose to evaluate the effects of 
clodronate-liposome treatment by flow cytometry. In the present study, macrophages were tentatively defined as cells with a high FSC/SSC and double negative for WCI-12 (recognizing surface Ig) and for TCL-BE8 (recognizing neutrophilic granulocytes).

The majority (approximately 65\%) of the resident peritoneal leukocyte population of naïve carp were macrophages. Intraperitoneal injection of liposomes alone induced a rapid $(<1 \mathrm{~d})$ redistribution of neutrophilic granulocytes from the head kidney to the peritoneal cavity. During the following $4 \mathrm{~d}$, the head kidney was repopulated with young neutrophils (different FSC/SSC profile). In other fish species as well, such as rainbow trout Oncorhynchus mykiss and goldfish Carassius auratus, macrophages are the resident phagocytes of the peritoneal cavity while significant numbers of neutrophils are present in the body cavity only during inflammatory responses (i.p. injection with bacteria or thioglycollate) (Afonso et al. 1998, Bielek et al. 1999).

Although i.p.-administered DiI-liposomes were apparently taken up by both neutrophilic granulocytes and macrophages, macrophages took up more liposomes per cell, as shown by a significantly higher fluorescence intensity. Thus, also in fishes, liposomes are primarily taken up by macrophages. Further, injections with clodronate-liposomes significantly affected peritoneal macrophages, as shown by a reduced uptake of fluorescent (DiI) liposomes after clodronateliposome treatment. A slight decrease in uptake of DiIliposomes after clodronate treatment was observed in the liver also. These observations indicate a succesful depletion of at least peritoneal macrophages in carp treated with clodronate-liposomes.

Application of the macrophage depletion model had a marginal (negative) effect on the removal of 'tryps' from the bloodstream only. Moribund carp did not show lethal parasitaemia but did suffer from systemic bacterial infections (in Trypanoplasma borreli-infected carp). Although we did not quantify the bacteraemia, this apparently caused heavy mortality. Heavy bacteraemia was also observed in fish from the non-infected but clodronate-liposome-treated group. Thus, lowered resistance to opportunistic bacteria was apparently related to a successful macrophage depletion but not to co-infection with parasites.

Successful depletion of (liver and peritoneal) macrophages seemed to have a minor effect on the resistance of carp to blood flagellates. Earlier studies have reported substantial phagocytosis of blood flagellates by fish peritoneal macrophages (Sypek \& Burreson 1983). Li \& Woo (1995) also found that the head kidney macrophages of rainbow trout could engulf 'tryps' after prior immunisation with a live attenuated form of Cryptobia (Trypanoplasma) salmositica. The method used in the above-mentioned studies, however, i.e. Giemsa-stained smears of leukocytes, could have mistaken adherence for phagocytosis. We found no evidence for substantial phagocytosis of $T$. borreli in electron-microscopic pictures of spleen, liver and head kidney of heavily infected carp (G.F. Wiegertjes \& J.P.J. Saeij unpubl. data). Also, Scharsack et al. (2003), using flow cytometry, found no evidence for phagocytosis of fluorochrome-labelled parasites. In addition, goldfish macrophages do not seem to phagocytose $T$. carassii (M. Belosevic pers. comm.). In the present study, successful depletion of the resident peritoneal macrophage population, induced by clodronate-liposome treatment, did not seem to lead to a significant increase in parasitaemia in either naïve or immune carp. Macrophages seem to have a minor effect on the resistance of carp against blood flagellates, but are essential as a first line of defence against bacterial infection.

Acknowledgements. We thank the central fish facilities, 'De Haar-Vissen' for providing and taking care of the carp. We also thank P. W. Van Tulden and Dr. N. Stockhofe, R. M. Buijs from the Central Institute for Animal Disease Control at Lelystad, The Netherlands, for facilitating the infection experiments and histological analyses, respectively. TCL-BE8 was a kind gift of Dr. N. Okamoto (Department of Aquatic Biosciences, Tokyo University of Fisheries, Tokyo, Japan), and Trypanosoma carassii was a kind gift of Dr. P. Overath, Max-Planck-Institut für Biologie, Abteilung Membranbiochemie, Tübingen, Germany. Clodronate was a gift from Roche Diagnostics. We further acknowledge the technical assistance of A. Taverne-Thiele and acknowledge Drs. J.H. W. M. Rombout, W. B. Van Muiswinkel and J. P. Scharsack for critically reading the manuscript. This research was supported by a grant from NWO-ALW (project 806-46.032-P) including a fellowship for J.P.J.S.

\section{LITERATURE CITED}

Afonso A, Lousada S, Silva J, Ellis AE, Silva MT (1998) Neutrophil and macrophage responses to inflammation in the peritoneal cavity of rainbow trout (Oncorhynchus mykiss). A light and electron microscopic cytochemical study. Dis Aquat Organ 34:27-37

Austin B, Austin DA (1987) Bacterial fish pathogens: disease in farmed and wild fish. Ellis-Horwood, Chichester

Barrow GI, Feltham RKA (1993) Cowan and Steel's manual for the identification of medical bacteria, 3rd edn. Cambridge University Press, Cambridge

Bielek E, Bigaj J, Chadzinska M, Plytycz B (1999) Depletion of head kidney neutrophils and cells with basophilic granules during peritoneal inflammation in the goldfish, Carassius auratus. Folia Biol (Krakow) 47:33-42

Biewenga J, Van der Ende MB, Krist LF, Borst A, Ghufron M, Van Rooijen N (1995) Macrophage depletion in the rat after intraperitoneal administration of liposome-encapsulated clodronate: depletion kinetics and accelerated repopulation of peritoneal and omental macrophages by administration of Freund's adjuvant. Cell Tissue Res 280: 189-196 
Bunnajirakul S, Steinhagen D, Hetzel U, Körting W, Drommer W (2000) A study of sequential histopathology of Trypanoplasma borreli (Protozoa: Kinetoplastida) in susceptible common carp Cyprinus carpio. Dis Aquat Org 39: 221-229

Espenes A, Press CM, Van Rooijen NV, Landsverk T (1997) Apoptosis in phagocytotic cells of lymphoid tissues in rainbow trout (Oncorhynchus mykiss) following administration of clodronate liposomes. Cell Tissue Res 289:323-331

Henson PM, Bratton DL, Fadok VA (2001) Apoptotic cell removal. Curr Biol 11:R795-805

Irnazarow I (1995) Genetic variability of Polish and Hungarian carp lines. Aquaculture 129:215-219

Jones SR, Palmen M, Van Muiswinkel WB (1993) Effects of inoculum route and dose on the immune response of common carp, Cyprinus carpio to the blood parasite, Trypanoplasma borreli. Vet Immunol Immunopathol 36:369-378

Krieg NR, Holt JG (eds) (1984) Bergey's manual of systematic bacteriology, Vol 1. Williams \& Wilkens, Baltimore, MD

Li S, Woo PT (1995) Efficacy of a live Cryptobia salmositica vaccine, and the mechanism of protection in vaccinated rainbow trout, Oncorhynchus mykiss, against cryptobiosis. Vet Immunol Immunopathol 48:343-353

Lom J, Dykova I (1992) Protozoan parasites of fishes. Elsevier, Amsterdam

Nakayasu C, Omori M, Hasegawa S, Kurata O, Okamoto N (1998) Production of a monoclonal antibody for carp (Cyprinus carpio L.) phagocytic cells and separation of the cells. Fish Shellfish Immunol 8:91-100

Overath P, Ruoff J, Stierhof YD, Haag J, Tichy H, Dyková I, Lom J (1998) Cultivation of bloodstream forms of Trypanosoma carassii, a common parasite of freshwater fish. Parasitol Res 84:343-347

Overath P, Haag J, Mameza MG, Lischke A (1999) Freshwater fish trypanosomes: definition of two types, host control by antibodies and lack of antigenic variation. Parasitology 119:591-601

Scharsack JP, Steinhagen D, Kleczka C, Schmidt JO, Körting W, Michael RD, Leibold W, Schuberth HJ (2003) Head kidney neutrophils of carp (Cyprinus carpio L.) are func-

Editorial responsibility: Wolfgang Körting,

Hannover, Germany tionally modulated by the haemoflagellate Trypanoplasma borreli. Fish Shellfish Immunol 14:389-403

Secombes CJ, Van Groningen JJ, Egberts E (1983) Separation of lymphocyte subpopulations in carp Cyprinus carpio L. by monoclonal antibodies: immunohistochemical studies. Immunology 48:165-175

Steinhagen D, Kruse P, Körting W (1989) The parasitemia of cloned Trypanoplasma borreli Laveran and Mesnil, 1901, in laboratory-infected common carp (Cyprinus carpio L.). J Parasitol 75:685-689

Sypek JP, Burreson EM (1983) Influence of temperature on the immune response of juvenile summer flounder, Paralichthys dentatus, and its role in the elimination of Trypanoplasma bullocki infections. Dev Comp Immunol 7: $277-286$

Van Rooijen N, Sanders A (1994) Liposome mediated depletion of macrophages: mechanism of action, preparation of liposomes and applications. J Immunol Methods 174:83-93

Van Rooijen N, Van Nieuwmegen R (1984) Elimination of phagocytic cells in the spleen after intravenous injection of liposome-encapsulated dichloromethylene diphosphonate. An enzyme-histochemical study. Cell Tissue Res 238: 355-358

Van Rooijen N, Van Nieuwmegen R, Kamperdijk EW (1985) Elimination of phagocytic cells in the spleen after intravenous injection of liposome-encapsulated dichloromethylene diphosphonate. Ultrastructural aspects of elimination of marginal zone macrophages. Virchows Arch B Cell Pathol Incl Mol Pathol 49:375-383

Van Rooijen N, Sanders A, Van den Berg TK (1996) Apoptosis of macrophages induced by liposome-mediated intracellular delivery of clodronate and propamidine. J Immunol Methods 193:93-99

Wiegertjes GF, Groeneveld A, Van Muiswinkel WB (1995) Genetic variation in susceptibility to Trypanoplasma borreli infection in common carp (Cyprinus carpio L.). Vet Immunol Immunpathol 47:153-161

Woo PTK (1979) Trypanoplasma salmositica: experimental infections in rainbow trout, Salmo gairdneri. Exp Parasitol $47: 36-48$

Submitted: March 31, 2003; Accepted: July 2, 2003

Proofs received from author(s): October 7, 2003 